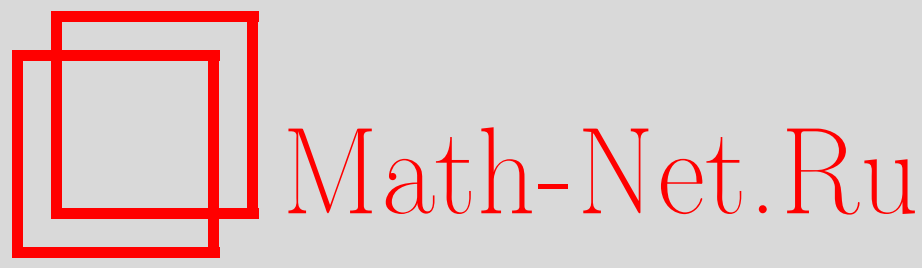

Г. В. Ефимов, Квантовая частица в случайной среде, ТМФ, 2015, том 185, номер 1, 86-98

DOI: https://doi.org/10.4213/tmf8902

Использование Общероссийского математического портала Math-Net.Ru подразумевает, что вы прочитали и согласны с пользовательским соглашением http://www . mathnet.ru/rus/agreement

Параметры загрузки :

IP : 54.224 .60 .19

26 апреля 2023 г., 13:26:59

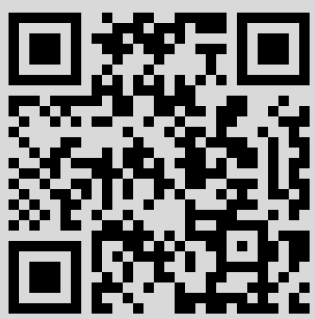




\title{
ФИЗИКА
}

Том 185, № 1

октябрь, 2015

(C) 2015 г.

Г. В. Ефимов*

\section{КВАНТОВАЯ ЧАСТИЦА В СЛУЧАЙНОЙ СРЕДЕ}

\begin{abstract}
Поведение квантовой частицы в случайной среде описывается функцией Грина в представлении функционального интеграла и предлагаются методы его оценивания. Вычислена длина локализации в случае движения в неупорядоченной среде и в среде, описывающейся случайным потенциалом гауссова типа. Рассмотрено движение квантовой частицы в вакууме квантованного поля. Показано различие между нерелятивистским и релятивистским подходами.
\end{abstract}

Ключевые слова: квантовая механика, случайные среды, длина локализации, функция Грина, функциональный интеграл, нерелятивистская и релятивистская динамика.

DOI: $10.4213 / \operatorname{tmf} 8902$

\section{1. ВВЕДЕНИЕ}

Проблема локализации квантовых состояний в неупорядоченной, или случайной среде изучается как теоретически, так и экспериментально, начиная с пионерской статьи Андерсена [1]. С тех пор немалое количество работ было посвящено тому, чтобы описать это явление исходя из уравнения Шредингера при разных предположениях относительно характера неупорядоченной среды (см., например, труды [2]-[7]).

Локализация - это эффект взаимодействия квантовой частицы со средой, приводящего к эффективному торможению частицы при движении в этой среде. Частица не теряет своей энергии, однако в процессе многократных упругих рассеяний на атомах среды она изменяет свою траекторию, т. е. не движется вдоль первоначального направления. Поток частиц вперед уменьшается. Другими словами, вследствие многократного рассеяния на неоднородностях и интерференции рассеянных волн становится невозможным распространение бегущих волн; колебания приобретают характер стоячей волны, сконцентрированной (локализованной) в ограниченной области пространства. Поэтому можно говорить об эффективном тормозящем эффекте случайной среды. Длина локализации является основной характеристикой неоднородной среды, характеризующей действие неоднородной среды на частицу. Задача теории - найти длину локализации при заданном характере случайной среды. Если характер случайности может быть так или иначе сформулирован на языке

* Лаборатория теоретической физики, Объединенный институт ядерных исследований, Дубна, Московская обл., Россия 
аналитических функций, то основным методом исследования является вычисление функции Грина частицы в среде на больших расстояниях.

Наша точка зрения состоит в том, что наиболее адекватным методом при вычислении функции Грина является метод функционального интегрирования. Для этого необходимо представить решение уравнения для соответствующей функции Грина в форме функционального интеграла. Однако это не всегда оказывается возможным. В частности, решение уравнения, предложенного в основополагающей работе Андерсена [1] и являющегося по сути интегро-дифференциальным, не удается представить в желаемом виде. Сам автор пользуется теорией возмущений, имея при этом проблему оценки $n$-го члена разложения. Если же представление в форме функционального интеграла получено, можно использовать методы, позволяющие выйти за рамки теории возмущений. Именно такие примеры рассмотрены в настоящей работе. В случае так называемой слабой локализации, когда случайные примеси малы, при вычислении функционального интеграла вполне применима теория возмущений.

В настоящей работе вычислены длины локализации в следующих наиболее типичных реализациях случайной среды:

1) среда представляет собой пространство, заполненное случайно расположенными атомами, частица, масса которой заметно меньше массы атома, взаимодействует с каждым атомом посредством заданного потенциала;

2) среда описывается случайным потенциалом с заданным распределением, типичным является гауссово распределение с заданной корреляционной функцией;

3) в роли случайного поля выступает квантованное поле, т. е. частица находится в вакууме квантованного поля, который играет роль случайной среды.

\section{2. ПОСТАНОВКА ЗАДАЧИ}

Квантовая частица в неупорядоченной, или случайной среде описывается гамильтонианом

$$
H=\frac{\mathbf{p}}{2 m}+W(\mathbf{x}) .
$$

Потенциал взаимодействия с неупорядоченной средой $W(\mathbf{x})$ описывает случайный характер взаимодействия частицы со средой. Относительно характера случайности делаются различные предположения.

Мы ищем стационарную функцию Грина во внешнем поле

$$
G_{k}\left(\mathbf{x}, \mathbf{x}^{\prime} \mid W\right)=\frac{1}{\mathbf{p}^{2} / 2 m+W(\mathbf{x})-k^{2} / 2 m+i 0} \delta\left(\mathbf{x}-\mathbf{x}^{\prime}\right),
$$

где $k$ - импульс частицы, находящейся в случайной среде. Затем надо усреднить функцию Грина по случайному потенциалу:

$$
G_{k}(r)=\left\langle G_{k}\left(\mathbf{x}, \mathbf{x}^{\prime} \mid W\right)\right\rangle_{W}, \quad \mathbf{r}=\mathbf{x}-\mathbf{x}^{\prime}, \quad r=|\mathbf{r}| .
$$

Длина локализации $\ell(k)=1 / \Gamma(k)$, зависящая от импульса частицы $k$, определяется поведением функции Грина на больших расстояниях:

$$
\frac{1}{\ell(k)}=\Gamma(k)=-\lim _{r \rightarrow \infty} \frac{\ln \left|G_{k}(r)\right|}{r}=-\lim _{r \rightarrow \infty} \frac{1}{r} \operatorname{Re} \ln G_{k}(r) .
$$

Задача состоит в том, чтобы вычислить предел в (4) в зависимости от характера случайности потенциала. 


\section{3. ФУНКЦИОНАЛЬНОЕ ПРЕДСТАВЛЕНИЕ}

Решение уравнения для функции Грина (2) можно представить в виде функционального интеграла, проводя стандартные преобразования (см., например, [8])

$$
\begin{aligned}
G_{k}\left(\mathbf{x}, \mathbf{x}^{\prime} \mid W\right)= & i \int_{0}^{\infty} d t \exp \left\{-i t\left[-\frac{1}{2 m} \frac{d^{2}}{d \mathbf{x}^{2}}+W(\mathbf{x})-\frac{\mathbf{k}^{2}}{2 m}-i 0\right]\right\} \delta\left(\mathbf{x}-\mathbf{x}^{\prime}\right)= \\
= & \int_{0}^{\infty} \frac{d t}{(2 \pi i t)^{3 / 2}} m^{3 / 2} \exp \left\{\frac{i}{2}\left[\frac{t \mathbf{k}^{2}}{m}+\frac{\left(\mathbf{x}-\mathbf{x}^{\prime}\right)^{2} m}{t}\right]\right\} \times \\
& \times \int \frac{D \boldsymbol{\eta}}{C} \exp \left\{i \int_{0}^{t} d \nu\left[\frac{m}{2} \dot{\boldsymbol{\eta}}^{2}(\nu)-W\left(\mathbf{x} \frac{\nu}{t}+\mathbf{x}^{\prime}\left(1-\frac{\nu}{t}\right)-\boldsymbol{\eta}(\nu)\right)\right]\right\}
\end{aligned}
$$

с краевыми условиями $\boldsymbol{\eta}(0)=\boldsymbol{\eta}(t)=0$.

Удобно перейти к безразмерным переменным. Для этого сделаем замены

$$
t=\frac{r m}{k} s, \quad \nu=\frac{v s}{k} \tau, \quad \mathbf{n}=\frac{\mathbf{r}}{r}, \quad \boldsymbol{\eta}(\nu)=\sqrt{\frac{s}{k}} \boldsymbol{\xi}(t),
$$

тогда

$$
\begin{aligned}
G_{k}\left(\mathbf{x}, \mathbf{x}^{\prime} \mid W\right)= & B \int_{0}^{\infty} \frac{d s}{s^{3 / 2}} \exp \left\{\frac{i r k}{2}\left(s+\frac{1}{s}\right)\right\} \times \\
& \times \int \frac{D \boldsymbol{\xi}}{C} \exp \left\{\frac{i}{2} \int_{0}^{r} d \tau\left[\dot{\boldsymbol{\xi}}^{2}(\tau)-i m \frac{s}{k} W\left(\mathbf{n} \tau+\mathbf{x}^{\prime}-\sqrt{\frac{s}{k}} \boldsymbol{\xi}(\tau)\right)\right]\right\} .
\end{aligned}
$$

По существу характер действия случайной среды на частицу определяется тем, как производится усреднение по случайному потенциалу $W$. А именно, требуется знать, к чему приводит усреднение

$$
\left\langle\exp \left\{-i m \frac{s}{k} \int_{0}^{r} d \tau W\left(\mathbf{n} \tau+\mathbf{x}^{\prime}-\sqrt{\frac{s}{k}} \boldsymbol{\xi}(\tau)\right)\right\}\right\rangle_{W}=e^{Z_{r}[s / k, \boldsymbol{\xi}]}
$$

Возникший после усреднения функциональный интеграл

$$
I_{r}\left(\frac{s}{k}\right)=\int \frac{D \boldsymbol{\xi}}{C} \exp \left\{\frac{i}{2} \int_{0}^{r} d \tau \dot{\boldsymbol{\xi}}^{2}(\tau)+Z_{r}\left[\frac{s}{k}, \boldsymbol{\xi}\right]\right\}
$$

можно рассматривать как функцию Грина частицы, движущейся в нелокальном потенциале. Таким образом, влияние случайной среды на частицу определяется нелокальным потенциалом $Z_{r}[s / k, \boldsymbol{\xi}]$. Заметим, что в силу трансляционной инвариантности функционал $Z_{r}[s / k, \boldsymbol{\xi}]$ не зависит от $\mathbf{x}^{\prime}$. Нас интересует поведение функции $I_{r}(s / k)$ при $r \rightarrow \infty$. В этом пределе получим

$$
I_{r}\left(\frac{s}{k}\right) \rightarrow C e^{r A(s / k)},
$$

где $C$ - некоторая константа, не представляющая для нас никакого интереса.

Окончательно для усредненной функции Грина получим представление в виде интеграла, который можно записать в двух формах:

$$
\begin{aligned}
G_{k}(r) & =B \int_{0}^{\infty} \frac{d s}{s^{3 / 2}} \exp \left\{r\left[\frac{i k}{2}\left(s+\frac{1}{s}\right)+A\left(\frac{s}{k}\right)\right]\right\}= \\
& =B \int_{0}^{\infty} \frac{d v}{v^{3 / 2}} \exp \left\{r\left[\frac{i k^{2}}{2} v+\frac{i}{2 v}+A(v)\right]\right\} .
\end{aligned}
$$


Точка перевала определяется из одного из двух эквивалентных уравнений

$$
\begin{array}{ll}
s_{\mathrm{c}}(k): & \frac{d}{d s}\left[\frac{i k}{2}\left(s+\frac{1}{s}\right)+A\left(\frac{s}{k}\right)\right]=0, \\
v_{\mathrm{c}}(k): & \frac{d}{d v}\left[\frac{i k^{2}}{2} v+\frac{i}{2 v}+A(v)\right]=0 .
\end{array}
$$

Тогда $G_{k}(r) \sim e^{r \Phi(k)}$, где

$$
\Phi(k)=\frac{i k}{2}\left(s_{\mathrm{c}}(k)+\frac{1}{s_{\mathrm{c}}(k)}\right)+A\left(\frac{s_{\mathrm{c}}(k)}{k}\right)=\frac{i k^{2}}{2} v_{\mathrm{c}}(k)+\frac{i}{2 v_{\mathrm{c}}(k)}+A\left(v_{\mathrm{c}}(k)\right) .
$$

В итоге для длины локализации получаем

$$
\frac{1}{\ell(k)}=\Gamma(k)=-\operatorname{Re} \Phi(k) .
$$

Представление функции Грина в двух формах (10) позволяет сделать качественный вывод о поведении длины локализации при больших и малых импульсах. При достаточно гладких корреляционных функциях можно ожидать, что функция $A(u)$ монотонно растет от нуля до бесконечности в пределах $0 \leqslant u \leqslant \infty$. Тогда при больших импульсах $k \rightarrow \infty$ в первом уравнении в (11) можно пренебречь слагаемым $A(s / k)$, в результате имеем

$$
\frac{d}{d s}\left[\frac{i k}{2}\left(s+\frac{1}{s}\right)\right]=0 \quad \Longrightarrow \quad s_{\mathrm{c}}=1
$$

и

$$
\operatorname{Re} \Phi(k) \rightarrow \operatorname{Re} A\left(\frac{1}{k}\right) \rightarrow 0
$$

При малых $k \rightarrow 0$ воспользуемся вторым уравнением, в котором можно пренебречь слагаемым с $k^{2}$, в результате имеем

$$
\frac{d}{d v}\left[\frac{i}{2 v}+A(v)\right]=0 \quad \Longrightarrow \quad v_{\mathrm{c}}=\text { const }
$$

и

$$
\operatorname{Re} \Phi(k) \rightarrow \operatorname{Re}\left[\frac{i}{2 v_{\mathrm{c}}}+A\left(v_{\mathrm{c}}\right)\right]=\text { const. }
$$

Отсюда можно заключить, что длина локализации конечна при малых импульсах и растет при больших импульсах.

Таким образом, задача состоит из следующих этапов:

- усреднение по случайному потенциалу (7);

- вычисление функционального интеграла (8) в пределе $r \rightarrow \infty$;

- определение точки перевала из уравнений (11).

Ниже мы рассмотрим перечисленные выше варианты случайной среды в рамках сформулированной схемы. 


\section{4. НЕУПОРЯДОЧЕННАЯ СРЕДА}

Неупорядоченная среда понимается как совокупность атомов, случайно расположенных в пространстве. Примерами таких сред являются аморфные металлы, металлические стекла или полупроводники с примесями. При этом считается, что массы атомов примеси много больше массы частицы, так что их можно рассматривать как неподвижные точки. Такая неупорядоченная среда описываются гамильтонианом, в котором потенциал взаимодействия квантовой частицы с атомами примеси может быть представлен в виде

$$
W(\mathbf{x})=W\left(\mathbf{x} \mid\left\{\mathbf{y}_{j}\right\}\right)=\sum_{j}^{N} V\left(\mathbf{x}-\mathbf{y}_{j}\right) .
$$

Здесь потенциал $V\left(\mathbf{x}-\mathbf{y}_{j}\right)$ описывает взаимодействие частицы, которая расположена в точке $\mathbf{x}$, с атомом примеси, находящимся в точке $\mathbf{y}_{j}$. Точки $\left\{\mathbf{y}_{j}\right\}$ являются случайными величинами с распределением

$$
P\left(\left\{\mathbf{y}_{j}\right\}\right)=\frac{1}{|\Lambda|^{N}}, \quad|\mathbf{y}| \leqslant|\Lambda|^{1 / 3}, \quad \mathbf{y} \in \Lambda \subset \mathbb{R}^{3} .
$$

При этом плотность считается заданной:

$$
n=\lim _{N,|\Lambda| \rightarrow \infty} \frac{N}{|\Lambda|}=\text { const. }
$$

Предполагается, что потенциал $V(\mathbf{x})$ является короткодействующим и может быть представлен в форме

$$
V(\mathbf{x})=g U\left(\frac{r}{R}\right), \quad|\mathbf{x}|=r .
$$

Здесь $g$ - константа связи, имеющая размерность энергии, $R$ - размер области действия потенциала. Безразмерная функция $U(s)$ сосредоточена в основном в области $s \leqslant 1$ и быстро убывает при $s \rightarrow \infty$. Будем предполагать, что эта функция нормирована условием $\int_{0}^{\infty} d s U(s)=1$.

Усреднение (7) по случайным координатам дает

$$
\begin{aligned}
e^{Z_{r}[s / k, \boldsymbol{\xi}]} & =\left\langle\exp \left\{-i m \frac{s}{k} \int_{0}^{r} d \tau W\left(\mathbf{n} \tau+\mathbf{x}^{\prime}-\sqrt{\frac{s}{k}} \boldsymbol{\xi}(\tau) \mid\left\{\mathbf{y}_{j}\right\}\right)\right\}\right\rangle_{W}= \\
& =\exp \left\{n \int d \mathbf{y}\left(\exp \left[-i m \frac{s}{k} \int_{0}^{r} d \tau V\left(\mathbf{y}+\mathbf{n} \tau-\sqrt{\frac{s}{k}} \boldsymbol{\xi}(\tau)\right)\right]-1\right)\right\}
\end{aligned}
$$

Задача сводится к вычислению функционального интеграла

$$
\begin{aligned}
e^{r A(s / k)}= & \int \frac{D \boldsymbol{\xi}}{C} \exp \left(\frac{i}{2} \int_{0}^{r} d \tau \dot{\boldsymbol{\xi}}^{2}(\tau)+\right. \\
& \left.+n \int d \mathbf{y}\left[\exp \left\{-i m \frac{s}{k} \int_{0}^{r} d \tau V\left(\mathbf{y}+\mathbf{n} \tau-\sqrt{\frac{s}{k}} \boldsymbol{\xi}(\tau)\right)\right\}-1\right]\right) .
\end{aligned}
$$


4.1. Случай малых плотностей. В представлении (18) интегрирование по $d \mathbf{y}$ ограничено областью $|\mathbf{y}| \leqslant R$ действия потенциала. Поэтому эффективной константой связи является параметр

$$
\mathrm{N}=n R^{3} \sim\left(\frac{R}{L}\right)^{3} \ll 1,
$$

где $L$ - среднее расстояние между атомами в неупорядоченной среде. Этот параметр во всех известных средах очень мал, и поэтому вполне оправданно разложение по этому параметру. Имеем при $(r \rightarrow \infty)$

$$
\begin{aligned}
r A\left(\frac{s}{k}\right) \approx n \int \frac{D \boldsymbol{\xi}}{C} \exp \left\{\frac{i}{2} \int_{0}^{r} d \tau \dot{\boldsymbol{\xi}}^{2}(\tau)\right\} \times \\
\quad \times \int d \mathbf{y}\left[\exp \left\{-i m \frac{s}{k} \int_{0}^{r} d \tau V\left(\mathbf{y}+\mathbf{n} \tau-\sqrt{\frac{s}{k}} \boldsymbol{\xi}(\tau)\right)\right\}-1\right]= \\
=-i r \cdot n m \frac{s}{k} \int d \mathbf{y} V(\mathbf{y}) \times \\
\quad \times \lim _{r \rightarrow \infty} \int \frac{D \boldsymbol{\xi}}{C} \exp \left\{\frac{i}{2} \int_{0}^{r} d \nu \dot{\boldsymbol{\xi}}^{2}(\nu)\right\} \exp \left\{-i m \frac{s}{k} \int_{0}^{r} d \tau V\left(\mathbf{y}+\mathbf{n} \tau-\sqrt{\frac{s}{k}} \boldsymbol{\xi}(\nu)\right)\right\} .
\end{aligned}
$$

Как показано в работе [8], амплитуда упругого рассеяния вперед на потенциале $V$ при импульсе $k / s$ определяется интегралом

$$
\begin{aligned}
f\left(\frac{k}{s}, 0\right)= & -\frac{m}{2 \pi} \int d \mathbf{y} V(\mathbf{y}) \times \\
& \times \int \frac{D \boldsymbol{\xi}}{C} \exp \left\{\frac{i}{2} \int_{0}^{\infty} d \tau \dot{\boldsymbol{\xi}}^{2}(\tau)\right\} \exp \left\{-i \frac{m s}{k} \int_{0}^{\infty} d \tau V\left(\mathbf{y}+\mathbf{n} \tau-\sqrt{\frac{s}{k}} \boldsymbol{\xi}(\tau)\right)\right\} .
\end{aligned}
$$

Таким образом, имеем

$$
A\left(\frac{s}{k}\right)=i \frac{r n}{2} \cdot 4 \pi \frac{s}{k} f\left(\frac{k}{s}, 0\right) .
$$

Амплитуду $f(k / s, 0)$ представим в форме

$$
i 4 \pi \frac{s}{k} f\left(\frac{k}{s}, 0\right)=i 4 \pi \frac{s}{k} \operatorname{Re} f\left(\frac{k}{s}, 0\right)-4 \pi \frac{s}{k} \operatorname{Im} f\left(\frac{k}{s}, 0\right)=i \phi\left(\frac{k}{s}\right)-\sigma\left(\frac{k}{s}\right),
$$

поскольку согласно условию унитарности полное сечение упругого рассеяния при импульсе $k / s$ равно

$$
\sigma\left(\frac{k}{s}\right)=4 \pi \frac{s}{k} \operatorname{Im} f\left(\frac{k}{s}, 0\right) .
$$

В случае малой плотности и при достаточно больших импульсах $k$ точка перевала определяется уравнением

$$
\frac{d}{d s}\left[\frac{i k}{2}\left(s+\frac{1}{s}\right)+i n \cdot 2 \pi \frac{s}{k} f\left(\frac{k}{s}, 0\right)\right]=0
$$

и реализуется при $s=1$. В итоге имеем

$$
\frac{1}{\ell(k)}=\Gamma(k)=-\operatorname{Re} \Phi(k)=n \sigma(k) .
$$


Падающий на среду со случайными примесями поток частиц убывает по хорошо известному закону

$$
I(r) \sim\left|G_{E}(r)\right|^{2} \sim e^{-r n \sigma(k)},
$$

где $\sigma(k)$ - полное сечение рассеяния. Полученная формула точно совпадает с известной феноменологической формулой, которая следует из постулата, что интенсивность $I(r)$ проходящего сквозь среду потока частиц уменьшается в соответствии с уравнением

$$
d I(r)=-n \sigma(k) I(r) d r \quad \Longrightarrow \quad I(r)=I_{0} e^{-n \sigma(k) r},
$$

где $n$ - плотность частиц вещества, $\sigma(k)$ - полное сечение рассеяния падающей частицы на частице вещества. Длина локализации

$$
\ell(k)=\frac{1}{n \sigma(k)}
$$

является длиной свободного пробега.

4.2. Случай малых импульсов. Феноменологическое уравнение (25) предполагает, что в неупорядоченной среде преобладает однократное рассеяние. Однако в пределе малых импульсов, когда "размер" частицы $\lambda \sim \hbar / k$ становится порядка расстояния между центрами примесей $L \sim(1 / n)^{1 / 3}$, уравнение $(25)$ не имеет места, и начинает играть роль многократное рассеяние.

Большие и малые импульсы можно разделить следующей оценкой. Величина

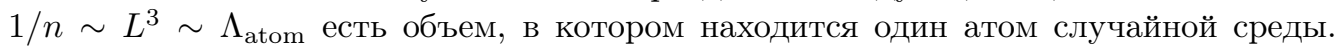
Произведение квадрата "размера" частицы на длину рассеяния $a / k^{2} \sim \lambda^{2} a \sim \Lambda_{\text {int }}$ можно считать объемом, где присходит взаимодействие частицы с атомом. Тогда

$$
\begin{aligned}
& \Lambda_{\text {int }} \ll \Lambda_{\text {atom }}, \quad \frac{a}{k^{2}} \ll \frac{1}{n} \quad \Longrightarrow \quad \text { большие импульсы } \\
& \Lambda_{\text {int }} \gg \Lambda_{\text {atom }}, \quad \frac{a}{k^{2}} \gg \frac{1}{n} \quad \Longrightarrow \quad \text { малые импульсы. }
\end{aligned}
$$

На языке уравнения (22) это означает, что при малых $k$ точка перевала по $s$ уже не реализуется при $s=1$. Поэтому для оценки длины локализации в этом случае в уравнении (22) надо представить амплитуду упругого рассеяния при малых импульсах как

$$
4 \pi \frac{s}{k} f\left(\frac{k}{s}, 0\right)=4 \pi \frac{s}{k} \operatorname{Re} f\left(\frac{k}{s}, 0\right)+i \sigma\left(\frac{k}{s}\right) \rightarrow 4 \pi \frac{s}{k} a+i \sigma\left(\frac{k}{s}\right),
$$

где $a$ - длина рассеяния. Тогда имеем

$$
\frac{i k}{2}\left(s+\frac{1}{s}\right)+i n \cdot 2 \pi \frac{s}{k} f\left(\frac{k}{s}, 0\right)=i\left[\frac{k}{2}\left(s+\frac{1}{s}\right)+\frac{2 \pi n a}{k} s\right]-n \sigma\left(\frac{k}{s}\right) .
$$

Поскольку при малых импульсах $\sigma(k / s) \sim a^{2} \ll(a / k)$, точку перевала найдем из уравнения

$$
\frac{d}{d s}\left[s\left(1+\frac{4 \pi n a}{k^{2}}\right)+\frac{1}{s}\right]=0
$$

имеем

$$
s_{\mathrm{c}}(k)=\frac{k}{\sqrt{k^{2}+4 \pi n a}} \approx \frac{k}{\sqrt{4 \pi n a}} .
$$


Для длины локализации получаем при малых импульсах

$$
\frac{1}{\ell(k)}=\Gamma(k)=-\operatorname{Re} \Phi(k)=n \sigma\left(\sqrt{k^{2}+4 \pi n a}\right) \rightarrow n \sigma(\sqrt{4 \pi n a}) .
$$

Полученная формула является оценкой длины свободного пробега при учете многократного рассеяния.

\section{5. ЧАСТИЦА В СЛУЧАЙНОМ ПОТЕНЦИАЛЕ}

Неупорядоченную среду можно феноменологически описать, предположив, что потенциал $W(\mathbf{x})$ является случайной функцией, например, гауссова типа с корреляционной функцией

$$
\langle W(\mathbf{x}) W(\mathbf{y})\rangle_{W}=K(\mathbf{x}-\mathbf{y})=g^{2} U\left(\frac{|\mathbf{x}-\mathbf{y}|}{R}\right),
$$

где $R$ - длина корреляции, а $g^{2}$ - константа “связи”, описывающая степень влияния примесей.

Проведем усреднение по $W$ :

$$
\begin{aligned}
e^{Z_{r}[k / s, \boldsymbol{\xi}]} & =\left\langle\exp \left\{-i \frac{m s}{k} \int_{0}^{r} d \tau W\left(\mathbf{n} \tau+\mathbf{x}^{\prime}-\sqrt{\frac{s}{k}} \boldsymbol{\xi}(\tau)\right)\right\}\right\rangle_{W}= \\
& =\exp \left\{-\frac{m^{2} s^{2}}{2 k^{2}} \iint_{0}^{r} d \tau d \tau^{\prime} K\left(\mathbf{n}\left(\tau-\tau^{\prime}\right)-\sqrt{\frac{s}{k}}\left(\boldsymbol{\xi}(\tau)-\boldsymbol{\xi}\left(\tau^{\prime}\right)\right)\right)\right\} .
\end{aligned}
$$

Слабая локализация. Если считать, что случайные добавки малы, а импульс $k$ велик, то можно воспользоваться теорией возмущений. Это так называемый случай слабой локализации. Тогда точка перевала при взятии интеграла по $s$ реализуется при $s=1$. После простых вычислений имеем

$$
\begin{aligned}
\frac{1}{\ell(k)}=\Gamma(k) & =-\operatorname{Re} A(k, 1)= \\
& =\frac{m^{2} \pi}{k} \int \frac{d \mathbf{p}}{(2 \pi)^{2}} \widetilde{K}(p) \delta\left(-\mathbf{p k}+\mathbf{p}^{2}\right)=\frac{m^{2}}{4 \pi k^{2}} \int_{0}^{k} d p p \widetilde{K}(p) .
\end{aligned}
$$

Подчеркнем, что это приближение справедливо при достаточно больших импульсах.

\section{6. ПРИБЛИЖЕНИЕ ПРЯМОЛИНЕЙНЫХ ПУТЕЙ}

Если импульс мал, то теорией возмущений воспользоваться нельзя, и задача состоит в том, чтобы получить разумное приближение для интеграла

$$
\begin{aligned}
& e^{r A(k / s)}=\int \frac{D \boldsymbol{\xi}}{C} \times \\
& \quad \times \exp \left\{\frac{i}{2} \int_{0}^{r} d \tau \dot{\boldsymbol{\xi}}^{2}(\tau)-\frac{m^{2} s^{2}}{2 k^{2}} \iint_{0}^{r} d \tau d \tau^{\prime} K\left(\mathbf{n}\left(\tau-\tau^{\prime}\right)-\sqrt{\frac{s}{k}}\left(\boldsymbol{\xi}(\tau)-\boldsymbol{\xi}\left(\tau^{\prime}\right)\right)\right)\right\} .
\end{aligned}
$$


Наша аргументация состоит в следующем. При больших $r$ основной вклад в интеграл дают функциональные переменные $\boldsymbol{\xi}(\tau)$ при больших $\tau$. Поскольку функциональный интеграл зависит от $r$ как линейная экспонента, следует ожидать, что при больших $\tau$ функциональная переменная линейно зависит от $\tau$, т. е. $\boldsymbol{\xi}(\tau) \sim \tau$. Сделаем в выписанном выше интеграле замену переменной интегрирования

$$
\boldsymbol{\xi}(\tau)=\mathbf{b} \tau+\boldsymbol{\eta}(\tau)
$$

где $\boldsymbol{\eta}(\tau)$ описывает флуктуации относительно прямолинейного пути. Тогда

$$
\begin{aligned}
e^{r A(s / k)}= & \int \frac{d \mathbf{b}}{C_{b}} e^{i r \mathbf{b}^{2} / 2} \int \frac{D \boldsymbol{\eta}}{C} \exp \left\{\frac{i}{2} \int_{0}^{r} d \tau \dot{\boldsymbol{\eta}}^{2}(\tau)\right\} \times \\
& \times \exp \left\{-\frac{m^{2} s^{2}}{2 k^{2}} \iint_{0}^{r} d \tau d \tau^{\prime} K\left(\left(\mathbf{n}+\mathbf{b} \sqrt{\frac{s}{k}}\right)\left(\tau-\tau^{\prime}\right)-\sqrt{\frac{s}{k}}\left(\boldsymbol{\eta}(\tau)-\boldsymbol{\eta}\left(\tau^{\prime}\right)\right)\right)\right\} .
\end{aligned}
$$

Исходя из вышесказанного пренебрежем флуктуациями $\boldsymbol{\eta}(\tau)$, в результате получим обычный интеграл

$$
\begin{aligned}
e^{r A(s / k)} & \approx \int \frac{d \mathbf{b}}{C_{b}} \exp \left\{\frac{i}{2} r \mathbf{b}^{2}-\frac{m^{2} s^{2}}{2 k^{2}} \iint_{0}^{r} d \tau d \tau^{\prime} K\left(\left(\mathbf{n}+\mathbf{b} \sqrt{\frac{s}{k}}\right)\left(\tau-\tau^{\prime}\right)\right)\right\}= \\
& =\int \frac{d \mathbf{b}}{C_{b}} \exp \left\{r\left(\frac{i}{2} \mathbf{b}^{2}-\frac{s^{2}}{k^{2}} \frac{G}{|\mathbf{n}+\sqrt{s / k} \mathbf{b}|}\right)\right\},
\end{aligned}
$$

где $G=m^{2} \int_{0}^{\infty} d \nu K(\mathbf{n} \nu)$. Этот интеграл при $r \rightarrow \infty$ вычисляется методом перевала, т. е. надо найти точку перевала следующей функции от $b=|\mathbf{b}|$ :

$$
\frac{i}{2} \mathbf{b}^{2}-\frac{s^{2}}{k^{2}} \frac{G}{|\mathbf{n}+\sqrt{s / k} \mathbf{b}|}=\frac{k}{s}\left[\frac{i}{2}(b-1)^{2}-\frac{s^{3}}{k^{3}} \frac{G}{b}\right] .
$$

В итоге в результате вычислений для длины локализации имеем

$$
\frac{1}{\ell(k)}=\Gamma(k)= \begin{cases}O\left(G / k^{2}\right), & k \rightarrow \infty \\ O\left(G^{1 / 3}\right), & k \rightarrow 0 .\end{cases}
$$

Длина локализации конечна при нулевом импульсе и плавно убывает при $k \rightarrow \infty$.

\section{7. ЧАСТИЦА В ВАКУУМЕ КВАНТОВАННОГО ПОЛЯ}

В формализме квантовой теории поля окружающая нас среда представляет собой вакуум всех возможных квантованных полей. И в этом вакууме невзаимодействующие частицы движутся без какого-либо тормозящего эффекта. С другой стороны, движение в вакуумных полях можно рассматривать как движение в случайной среде. Возникает вопрос, чем отличается квантово-полевой вакуум от квантовомеханической среды со случайным потенциалом? Другими словами, что будет происходить с квантовой частицей, если этим случайным полем будет квантованное поле? Проблему можно сформулировать следующим образом. Квантовая частица взаимодействует с некоторым квантованным полем и находится в вакууме этого 
поля. С формальной точки зрения квантованное поле выступает в роли гауссова случайного поля со вполне определенной корреляционной функцией. Может ли это взаимодействие привести к тормозящему эффекту, т. е. отлична ли от нуля длина локализации при движении квантовой частицы в вакууме квантованного поля?

Возможны две точки зрения.

1. Согласно первой из этих точек зрения квантованное поле $\Phi(\mathbf{x})$ рассматривается как внешнее гауссово случайное поле со своей специфической корреляционной функцией. Гамильтониан квантовой частицы в этом случае имеет вид

$$
H=\frac{\mathbf{p}^{2}}{2 m}+h \Phi(\mathbf{x}) .
$$

Движение частицы в этом квантованном случайном поле описывается усредненной функцией Грина

$$
G_{k}(\mathbf{x}-\mathbf{y})=\left\langle\frac{1}{\mathbf{p}^{2} / 2 m+h \Phi(\mathbf{x})-\mathbf{k}^{2} / 2 m} \delta(\mathbf{x}-\mathbf{y})\right\rangle_{\Phi} .
$$

При такой постановке задачи условие, что поле $\Phi(\mathbf{x})$ является квантованным полем, сводится лишь к тому, что корреляционная функция имеет определенный вид:

$$
K(\mathbf{x}-\mathbf{y})=h^{2}\langle\Phi(\mathbf{x}) \Phi(\mathbf{y})\rangle_{\Phi}
$$

где усреднение проводится по вакууму квантованного поля.

2. Другая точка зрения состоит в том, что должна рассматриваться квантовая система, состоящая из квантовой частицы и квантованного поля. Гамильтониан при такой постановке задачи выглядит несколько иначе:

$$
H_{\mathrm{tot}}=H_{0}+H=H_{0}+\frac{\mathbf{p}^{2}}{2 m}+h \Phi(\mathbf{x}),
$$

где следует добавить гамильтониан $H_{0}$, описывающий вакуум квантованного поля, так что функция Грина частицы равна

$$
G_{k}(\mathbf{x}-\mathbf{y})=\left\langle\frac{1}{H_{0}+\mathbf{p}^{2} / 2 m+h \Phi(\mathbf{x})-\mathbf{k}^{2} / 2 m} \delta(\mathbf{x}-\mathbf{y})\right\rangle_{\Phi} .
$$

Здесь усреднение проводится по вакууму квантованного поля.

Как мы увидим ниже, усреднение в (36) и (39) приводит к различным результатам, хотя и в том, и в другом случае корреляционная функция, казалось бы, одна и та же.

7.1. Поле. Для простоты будем рассматривать однокомпонентное скалярное поле $\Phi(\mathbf{x})$ с массой $\mu$. Для свободного гамильтониана и поля имеем

$$
\begin{gathered}
H_{0}=\int d \mathbf{p} \omega(p) a_{\mathbf{p}}^{+} a_{\mathbf{p}}, \quad \omega(\mathbf{p})=\sqrt{\mathbf{p}^{2}+\mu^{2}} \\
\Phi(\mathbf{x}, t)=\int \frac{d \mathbf{p}}{(2 \pi)^{3 / 2} \sqrt{2 \omega(\mathbf{p})}}\left(a_{\mathbf{p}} e^{-i \omega(\mathbf{p}) t+i \mathbf{p x}}+a_{\mathbf{p}}^{+} e^{i \omega(\mathbf{p}) t^{\prime}-i \mathbf{p x}}\right) .
\end{gathered}
$$

Будем пользоваться обозначениями

$$
\Phi(\mathbf{x})=\Phi(\mathbf{x}, 0), \quad \Phi(\mathbf{x}, t)=e^{i H_{0} t} \Phi(\mathbf{x}) e^{-i H_{0} t} .
$$


Вакуум скалярного поля $|0\rangle$ определен как $a_{\mathbf{k}}|0\rangle=0$. Корреляционная функция поля имеет вид

$$
\begin{aligned}
&\langle 0| \mathrm{T}\left\{\Phi(\mathbf{x}, t) \Phi\left(\mathbf{x}^{\prime}, t^{\prime}\right)\right\}|0\rangle=D_{\mathrm{c}}\left(\mathbf{x}-\mathbf{x}^{\prime}, t-t^{\prime}\right)= \\
& \quad=\int \frac{d \mathbf{p}}{(2 \pi)^{3} 2 \omega(\mathbf{p})} e^{-i \omega(\mathbf{p})\left|t-t^{\prime}\right|+i \mathbf{p}\left(\mathbf{x}-\mathbf{x}^{\prime}\right)}=\int \frac{d^{4} p}{i(2 \pi)^{4}\left(-p^{2}+\mu^{2}-i 0\right)} e^{i p\left(x-x^{\prime}\right),}
\end{aligned}
$$

где $p^{2}=p_{0}^{2}-\mathbf{p}^{2}$. При равных временах $t=t^{\prime}$ имеем

$$
\left\langle 0\left|\Phi(\mathbf{x}) \Phi\left(\mathbf{x}^{\prime}\right)\right| 0\right\rangle=D\left(\mathbf{x}-\mathbf{x}^{\prime}\right)=\int \frac{d \mathbf{p}}{(2 \pi)^{3} 2 \omega(\mathbf{p})} e^{i \mathbf{p}\left(\mathbf{x}-\mathbf{x}^{\prime}\right)} .
$$

7.2. Нерелятивистское уравнение. Повторяя изложенную выше схему вычислений, получим для усредненной функции Грина

$$
\begin{aligned}
& G_{k}(r)=\left\langle 0\left|\frac{1}{\mathbf{p}^{2} / 2 m+h \Phi(\mathbf{x})-\mathbf{k}^{2} / 2 m} \delta(\mathbf{x}-\mathbf{y})\right| 0\right\rangle= \\
& =B \int_{0}^{\infty} \frac{d s}{s^{3 / 2}} \exp \left\{\frac{i}{2} k r\left(s+\frac{1}{s}\right)\right\} \times \\
& \quad \times \int \frac{D \boldsymbol{\xi}}{C} \exp \left\{\frac{i}{2} \int_{0}^{r} d \tau \dot{\boldsymbol{\xi}}^{2}(\tau)-\frac{g^{2} s^{2}}{2 k^{2}} \iint_{0}^{r} d \tau d \tau^{\prime} D\left(\mathbf{n}\left(\tau-\tau^{\prime}\right)-\sqrt{\frac{s}{k}}\left(\boldsymbol{\xi}(\tau)-\boldsymbol{\xi}\left(\tau^{\prime}\right)\right)\right)\right\} .
\end{aligned}
$$

Здесь введена константа $g=2 m h$.

Если константа связи $g$ достаточно мала, а импульс $k$ велик, то справедлива теория возмущений, т. е. имеет место слабая локализация. В этом случае точка перевала реализуется при $s=1$, и мы имеем при больших $r$

$$
\frac{1}{\ell(k)}=\Gamma(k)=-\frac{1}{r} \operatorname{Re} \ln G_{k}(r)=\frac{g^{2}}{8 \pi} \frac{\sqrt{\mu^{2}+k^{2}}-\mu}{k^{2}} .
$$

Таким образом, длина локализации отлична от нуля при любых импульсах. Это означает, что при такой постановке задачи вакуум всегда оказывает тормозящее действие на движущуюся квантовую частицу.

Рассмотрим функцию Грина во втором случае. В тех же обозначениях и используя ту же технику вычислений, получим для длины локализации

$$
\frac{1}{\ell(k)}=\Gamma(k)=\frac{g^{2}}{k^{2}} \int_{0}^{\infty} \frac{d p p}{(2 \pi)^{2} 2 \omega(p)} \theta\left(\frac{k}{m}-\left(\sqrt{1+\frac{\mu^{2}}{p^{2}}}+\frac{p}{m}\right)\right)
$$

И

$$
\frac{1}{\ell(k)}=\Gamma(k)=0 \quad \text { при } \quad \frac{k}{m}<\min _{p}\left(\sqrt{1+\frac{\mu^{2}}{p^{2}}}+\frac{p}{m}\right) .
$$

Если $\mu=0$, то

$$
\frac{1}{\lambda(k)}=\Gamma(k)=\frac{g^{2} m^{2}}{k^{2}} \frac{k-m}{F} 8 \pi^{2} \theta(k-m) .
$$

Таким образом, оказалось, что при малых импульсах $k<m$ длина локализации бесконечна, т. е. нерелятивистская частица не тормозится. Но при $k>m$, т. е. в релятивистской области, торможение возникает, что явно противоречит реальности. Это означает, что нерелятивистское приближение имеет естественное ограничение. Другими словами, при больших импульсах необходимо учитывать релятивистский характер движения. 


\section{8. РЕЛЯТИВИСТСКОЕ УРАВНЕНИЕ}

Как говорилось выше, в релятивистской квантовой теории поля стабильная частица, находящаяся в вакууме других квантованных полей, движется как обычная свободная частица без какого-либо противодействия этого вакуума. В стандартном формализме квантовой теории поля, использующем теорию возмущений, это обеспечивается тем, что квантово-полевая функция Грина частицы имеет простой полюс в точке $k^{2}=m_{\mathrm{r}}^{2}$. Результат взаимодействия с вакуумом сводится только к перенормировке массы частицы: $m_{\mathrm{r}}^{2}=m^{2}+\delta m^{2}$.

Покажем, как этот же результат получается в формализме уравнения для функции Грина, в котором взаимодействие с квантованным полем рассматривается как внешнее случайное поле. Рассмотрим релятивистское уравнение

$$
\left[\square_{x}+m^{2}+g \Phi(x)\right] G\left(x-x^{\prime}\right)=\delta\left(x-x^{\prime}\right),
$$

где $\square=\partial_{t}^{2}-\nabla^{2} R$ и $x^{2}=x_{0}^{2}-\mathbf{x}^{2}$. Усреднение функции Грина по вакууму квантованного поля дает

$$
\begin{aligned}
& G(x-y)=\left\langle\frac{1}{\square_{x}+m^{2}+g \Phi(x)} \delta(x-y)\right\rangle_{\Phi}= \\
& =B \int_{0}^{\infty} \frac{d s}{s^{3 / 2}} \exp \left\{-\frac{i}{2}\left(s m^{2}+\frac{(x-y)^{2}}{s}\right)\right\} \times \\
& \quad \times \int \frac{D \xi}{C} \exp \left\{-\frac{i}{2} \int_{0}^{s} d \tau \dot{\xi}^{2}(\tau)-\frac{g^{2}}{2} \iint_{0}^{s} d \tau d \tau^{\prime} D_{\mathrm{c}}\left((x-y) \frac{\tau-\tau^{\prime}}{s}+\xi(\tau)-\xi\left(\tau^{\prime}\right)\right)\right\} .
\end{aligned}
$$

В пределе $(x-y)^{2} \rightarrow \infty$ имеем

$$
\begin{aligned}
G(x-y) & =B \int_{0}^{\infty} \frac{d s}{s^{2}} \exp \left\{-\frac{i}{2}\left(s m^{2}+\frac{\left(x-x^{\prime}\right)^{2}}{s}\right)+i s M_{\Lambda}\left(\frac{(x-y)^{2}}{4 s^{2}}\right)\right\}= \\
& =B \int_{0}^{\infty} \frac{d s}{s^{2}} \exp \left\{-i\left|x-x^{\prime}\right|\left[\frac{1}{2}\left(s\left(m^{2}+g^{2} \ln \Lambda\right)+\frac{1}{s}\right]\right\} \rightarrow e^{-i m_{r}|x-y|},\right.
\end{aligned}
$$

где

$$
M_{\Lambda}\left(\frac{(x-y)^{2}}{4 \mu^{2}}\right)=\frac{g^{2}}{2} \int_{0}^{1} d \alpha \operatorname{reg} \int \frac{d^{4} p_{E}}{(2 \pi)^{4}\left(p_{E}^{2}+\alpha^{2} z^{2} / \mu^{2}+(1-\alpha)\right)^{2}} .
$$

В итоге квантово-полевой вакуум не тормозит частицу, взаимодействие с вакуумом приводит лишь к перенормировке массы частицы, как это следует из стандартной квантовой теории поля. Таким образом, полученный результат еще раз подчеркивает самосогласованность представлений релятивизма и квантовой динамики частиц и указывает на пределы применимости представлений нерелятивистской квантовой механики.

\section{9. ЗАКЛЮЧЕНИЕ}

В настоящей работе продемонстрирована эффективность метода функционального интегрирования в задачах движения квантовой частицы в случайных средах. Получено обобщение известной формулы для длины свободного пробега в среде,

4 Теоретическая и математическая физика, т. 185, № 1, 2015 г. 
когда должно быть учтено многократное рассеяние. Предложен метод, названный приближением малых флуктуаций, для оценки длины локализации в случае больших неоднородностей. Рассмотрено движение квантовой частицы в вакууме квантованного поля. Оказалось, что имеет место следующее:

- если квантованное поле рассматривается как случайное поле со своей корреляционной функцией, то локализация происходит при любых импульсах;

- если квантованное поле является частью квантовой системы со своим гамильтонианом, то локализация происходит только при импульсах, больших массы частицы $(k>m)$, что означает наличие естественной границы применимости нерелятивистской квантовой механики;

- в случае релятивистского волнового уравнения, описывающего релятивистское движение в вакууме квантованного поля, локализация отсутствует.

Благодарности. В заключение приношу свою благодарность В. К. Лукьянову, Н. М. Плакиде, В. Б. Приезжеву, В. И. Юкалову за плодотворные обсуждения.

\section{Список литературы}

[1] P. W. Anderson, Phys. Rev., 109:5 (1958), 1492-1505.

[2] B. Kramer, A. MacKinnon, Rep. Progr. Phys., 56:12 (1993), 1469-1564.

[3] G. Bergman, Phys. Rep., 107:1 (1984), 1-58.

[4] В. Л. Бонч-Бруевич, И. П. Звягин, Р. Кайпер, А. Г. Миронов, Р. Эндерлайн, Б.-М. Эссер, Электронная теория неупорядоченных полупроводников, Наука, М., 1981.

[5] И. М. Лифшиц, С.А. Гредескул, Л.А. Пастур, Введение в теорию неупорядоченных систем, Наука, М., 1982.

[6] P. A. Lee, T. V. Ramakrishnan, Rev. Modern Phys., 57:2 (1985), 287-338.

[7] Н. А. Власов, Нейтронь, ГИТТЛ, М., 1955.

[8] Г. В. Ефимов, ТМФ, 179:3 (2014), 367-386. 